\title{
Development of Biomarkers Based on Diet-Dependent Metabolic Serotypes: Concerns and Approaches for Cohort and Gender Issues in Serum Metabolome Studies
}

\author{
UGO PAOLUCCI, ${ }^{1}$ KAREN E. VIGNEAU-CALLAHAN, ${ }^{2}$ HONGLIAN SHI, ${ }^{1}$ \\ WAYNE R. MATSON, ${ }^{2}$ and BRUCE S. KRISTAL ${ }^{3}$
}

\begin{abstract}
Mathematical models that reflect the effects of dietary restriction (DR) on the sera metabolome may have utility in understanding the mechanisms of DR and in applying this knowledge to human epidemiological studies. Previous studies demonstrated both the feasibility of identifying biomarkers through metabolome analysis and the validity of our approach in independent cohorts of 6-month-old male and female ad libitum fed or DR rats. Cross-cohort studies showed that cohort-specific effects distorted the dataset. The present study extends these observations across the entire sample set, thereby validating our markers independently of specific cohorts. Metabolites originally identified in males were examined in females and vice-versa. DR's effect on the metabolome is partially gender-specific and is modulated by environmental factors. DR reduces inter-gender differences in the metabolome. Univariate statistical methods showed that $56 / 93$ metabolites in the female samples and $39 / 93$ metabolites in the male samples were significantly altered (using our previous cut-off criteria of $p \leq 0.2$ ) by $D R$. The metabolites modulated by DR present a wide spectrum of concentration, redox reactivity and hydrophilicity, suggesting that our serotype is broadly representative of the metabolome and that DR has broad effects on the metabolome. These studies, coupled with those in the preceding and following reports, also highlight the utility for consideration of the metabolome as a network of metabolites using appropriate data analysis approaches. The inter-cohort and inter-gender differences addressed herein suggest potential cautions, and potential approaches, for identification of multivariate biomarker profiles that reflect changes in physiological status, such as a metabolism that predisposes to increased risk of neoplasia.
\end{abstract}

\section{INTRODUCTION}

T HE MAJOR IMPETUS for the investigation of dietary restriction (DR) in animal models stems from its favorable modulation of a wide variety of physiologic as well as pathologic processes, including life-

\footnotetext{
'Dementia Research Service, Burke Medical Research Institute, White Plains, New York.

${ }^{2}$ ESA, Inc., Chelmsford, Massachusetts.

${ }^{3}$ Departments of Biochemistry and Neuroscience, Cornell University Medical College, New York, New York.
} 


\section{PAOLUCCI ET AL.}

span and overall risk of certain cancers (Yu, 1994; Weindruch and Walford, 1988; Klurfield et al., 1987; Kritchevsky et al., 1984; Fernandes et al., 1995). The underlying effect of DR is thought to be attributable to a reduction in caloric intake as opposed to restriction of certain components in the diet (Iwasaki et al., 1988; Kristal and Yu, 1994; Maeda et al., 1985; Shimokawa et al., 1996). This leads to a broad overview hypothesis that DR modulates metabolism, and that this modulation is reflected in the sera metabolome. Thus, we have proposed that the metabolome will reflect the altered metabolism that must be occurring in these animals (Shi et al., 2002b,c; Vigneau-Callahan et al., 2001). We are therefore taking a data-driven approach toward characterizing the metabolome, with an eye towards using this work in the future to identify biomarkers both for cancer risk in individuals as well as for better understanding of the epidemiological relationships between caloric intake/balance and disease (Willett et al., 1999).

Our studies have therefore been directed toward the identification of serum metabolite profiles reflective of the changes associated with DR. Inherent to studies of this nature is the production of large quantities of data requiring the use of powerful multivariate statistical methods for analysis (Eriksson et al., 2001; Kennedy et al., 1998; Manly, 2000). Studies concerned with multi- or megavariate data acquisition generate one of the resources necessary for building powerful models for sample categorization. While, ideally and in theory, all data in a study are derived exactly equally, this rarely, if ever, occurs in practice, as both biological and analytical confounds can hamper direct comparisons between datasets, even within a single laboratory or study. We begin to address these issues in our study in the current report.

Our previous reports detailing the application of HPLC-based methods to measure low molecular weight redox-active metabolites in rodent sera have established both its feasibility and validity in determining possible serotypes indicative of the effects of DR (Shi et al., 2002b,c; Vigneau-Callahan et al., 2001; Shi et al., 2004). The present report seeks to continue this line of investigation by carrying out further analysis to maximize the number of useful metabolites while at the same time effectively eliminating any that are not informative or redundant. Related to this effort is the need to begin to compress inter-cohort variability (essentially experimental noise) that would otherwise obscure the signal of interest (AL-DR differences in the metabolome). One aspect of this requirement is the need to generate normalized, cross-cohort datasets. This requirement is highlighted by the difficulties encountered by SIMCA (Soft Independent Modeling of Class Analogy, a specific pattern recognition approach) when working (training) on single cohort training sets, as described in the accompanying report (Shi et al., 2004).

We further begin comparative analysis of how gender modulates the effects of DR on the metabolome. Despite the fact that the mechanisms of DR are not yet fully characterized, it is widely apparent that gender can play a significant role with respect to the physiological effects of DR (Yu, 1994). Therefore, it is reasonable to conclude that whatever the mechanism of DR may be, gender-based variability must be accounted for in order to fully comprehend its effects.

\section{MATERIALS AND METHODS}

\section{Animal husbandry}

Details of the rats and animal husbandry conditions used in this study have been reported previously (Vigneau-Callahan et al., 2001). Briefly, male and female Fischer 344 x Brown Norway $F_{1}$ rats were obtained monthly from the National Institute on Aging colony at Harlan (Indianapolis, IN) at 5 months of age and sacrificed approximately one month later. The basic animal husbandry for the animals used followed NIA/NIH guidelines as implemented by Harlan. Rats were fed NIH-31 (AL rats only) or vitamin-mineral fortified NIH-31 (DR rats only) (Vigneau-Callahan et al., 2001) for detailed diet compositions. All rats were individually housed and DR feeding regimens ( $40 \%$ less chow than eaten by AL rats) were implemented at 6 weeks of age. Animals were as follows: females (cohort A: 6 AL/6 DR rats; cohort B: 8 AL/7 DR rats; cohort $\mathrm{C}$ had $8 \mathrm{AL} / 8 \mathrm{DR}$ rats); and males (cohort A: $8 \mathrm{AL} / 8 \mathrm{DR}$ rats; cohort B: 7 AL/6 DR rats; cohort C: $8 \mathrm{AL} / 6 \mathrm{DR}$ rats). All animal experiments were performed under institutionally approved protocols and complied with the Guide for Care and Use of Laboratory Animals. 


\section{HPLC methodology}

HPLC separations and coulometric array detection was conducted essentially as described previously, using ESA CoulArray system (ESA, Inc., Chelmsford, MA) (Kristal et al., 1998, 2002; Matson et al., 1984, 1987; Shi et al., 2002a; Vigneau-Callahan et al., 2001).

\section{Statistical analysis}

Data analysis is described in the text. Data were analyzed using the programs CEAS 504 (ESA, Inc., Chelmsford, MA) and Statview 5.0.1 (SAS Institute, Cary, NC).

\section{RESULTS AND DISCUSSION}

In this report, we begin the process of making our sets of biomarkers more robust by conducting intercohort and inter-gender comparisons. This analysis thus builds on our earlier reports, where we focused on analysis of intra-cohort and intra-gender data.

These comparisons are expected to strengthen the overall profiles in several ways. Analysis of each original sample with its opposite-gender serotype (cross-gender analysis) may potentially serve to increase the number of useful redox-active metabolites under investigation and contribute to the overall predictive value of the serotypes. Combination of the data obtained from each cohort will allow us to reduce chromatogram variability through normalization against serum tyrosine concentration. Identification of chromatogram peaks corresponding to back-waves will allow elimination of non-informative data. Subsequent application of univariate statistical methods to the complete data set enables identification of metabolites that are potentially informative independent of cohort. Finally, we intend to apply principal component-based multivariate statistical methods on our data set in order to evaluate its potential use in the creation of a predictive model of DR.

\section{Initial cross-gender studies}

Integration and cross-comparisons of metabolites that comprise male and female serotypes allows identification of metabolites that may be useful in both genders. Initial analysis revealed twenty-five metabolites that were considered important for discrimination of the DR serotype in both males and females. This comparison enables us to define three groups of metabolites: (A) those metabolities apparently altered only in females; (B) those metabolites apparently altered only in males, and; (C) those metabolites apparently altered in both genders. In practical terms, the fact that male and female samples hold certain informative metabolites in common results in a reduction in the analyte set under investigation, since the final number will be significantly less than the sum of the two original sets. That is to say, the original 63 metabolites of the female serotype and the original 66 metabolites of the male serotype (Shi et al., 2002a,b) yield a combined serotype of 104 metabolites. Moreover, computer-based peak identification and quantification as well as eventual manual verification of sample chromatograms will also be facilitated since one chromatogram standard containing female, male and male/female serotypes can be used for analysis.

Cross-gender analysis additionally offers the potential to identify important metabolites that were missed in the first pass due to the analytical approaches taken for male and female samples (Shi et al., 2002b,c; Vigneau-Callahan et al., 2001). In theory, cross-gender analysis was to be conducted by direct quantitation, in all male samples, of those metabolites previously contained only in the female serotype, and, conversely, direct quantitation, in all female samples, of those metabolites previously contained only in the male serotype. In practice, we observed the requirement for intermediate steps.

\section{Metabolite hydrophobicity and electrochemical distribution}

Initial plots of previously identified metabolites across both retention time and electrochemical potential revealed two previously unappreciated aspects of our data (Fig. 1). It is apparent that the previously identified metabolites are widely distributed across both hydrophobicity (reflected in retention time) and elec- 


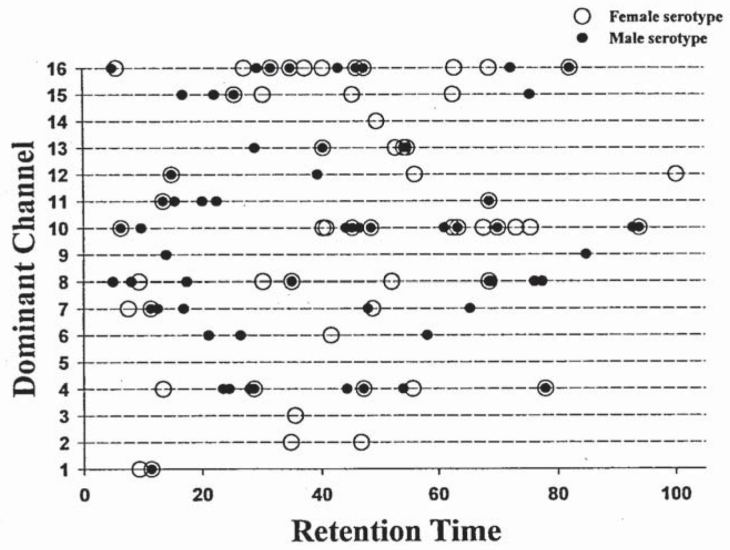

FIG. 1. Distribution of hydrophobicity and electrochemical reactivity of metabolites under investigation. The y-axis marks the dominant channel on which the particular metabolite gives most intense signal, while the $x$-axis marks the metabolite's retention time on that dominant channel. Solid circles represent metabolites that were originally defined in the male serotype, while empty circles represent metabolites that were originally defined in the female serotype. Overlapping points indicate metabolites shared by the two serotypes.

trochemical potential (reflected in distribution across dominant channel). We also noted that some metabolites contain multiple redox-active functional groups that display in a sample chromatogram as a primary wave in its low potential dominant and subdominant channels accompanied by its associated back-wave(s) in successive higher potential channels. In theory, back-waves are peaks from compounds that exhibit two or more oxidation events in the array because of their electrochemical properties and should reflect similar information about the variation of metabolite concentration as the primary wave. Treatment of back-waves was not a priority in previous studies, which were intrinsically driven by the desire to find peaks with utility in defining class membership. Backwaves are now addressed and, where appropriate, eliminated, to reduce the number of non-informative metabolites present in the combined serotype.

Elimination of redundant information contained in back-waves compresses the data set by decreasing the overall number of metabolites under investigation. This compression prevents multiple utilization of the same metabolite with subsequent over-weighting of the information contained therein. Primary waves and their accompanying back-waves were initially identified by visual inspection of the peaks contained in the standard chromatogram used for metabolite quantification. Subsequent verification consisted in the determination of the correlation coefficient ( $r$ ) of the primary wave and its suspected back-wave in order to determine the correlation between each. In the case of multiple suspected back-waves, $r^{2}$ values were calculated for all possible pairings of primary and back-waves, with subsequent evaluation of the correlation between the primary wave and the mean of all the waves (Fig. 2).

In the example presented in Figure 2, we observed a likely primary wave with a retention time of 6.408 $\mathrm{min}$, and its suspected back-waves at 6.425 and $6.467 \mathrm{~min}$, respectively. The fact that the pairings shown have such high $r^{2}$ values demonstrates the validity of the theory that the information contained in the backwaves can be represented by the primary wave alone. This analysis has permitted pruning of the components of the combined metabolite serotype by 11 to a total of 93 while minimizing any loss of potential predictive value. 


\section{DIET METABOLIC SEROTYPES-CROSS-COHORT STUDIES}

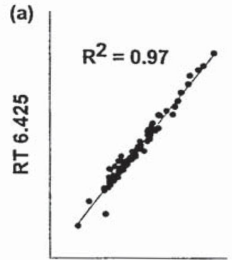

RT 6.408

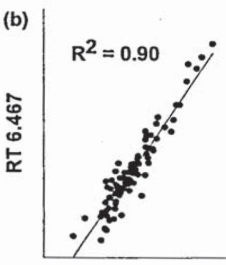

RT 6.408

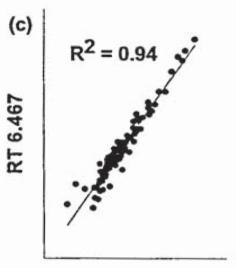

RT 6.425

(d)

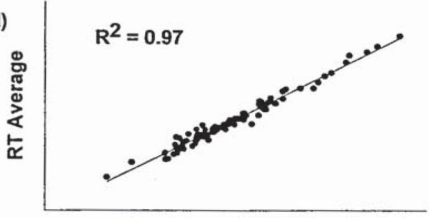

RT 6.408

FIG. 2. Example of back-wave elimination. Panels a, b, and c depict calculation of the $r^{2}$ values of all possible pairings of the primary wave with a retention time of $6.408 \mathrm{~min}$ (on its dominant channel) and its suspected back-waves at 6.425 and $6.467 \mathrm{~min}$, respectively. Graph d depicts the calculation of the $r^{2}$ value of the primary wave and the mean value of all three waves. The strong correlation between all possible pairings indicates that no loss of information will accompany elimination of peaks representing metabolites that are back-waves of other metabolites.

Metabolites were found to be broadly representative of a range of both concentrations (Fig. 3) as well as hydrophilicities and redox potentials (Fig. 1). The abscissa of Figure 3 represents the logarithm of the raw score of current (proportional to concentration) that each metabolite produces on its dominant channel in the chromatogram standard while the ordinate represents the number of metabolites with a current of that order of magnitude. Although the majority of metabolite peaks produce currents in the range of thousands and tens of thousands of instrument counts (arbitrary units), the peaks represent a fairly wide range of concentrations ( $\sim 33,000$-fold difference between the lowest and highest values).

\section{Cross-cohort normalization}

Normalization of quantified metabolite values based on tyrosine concentration in the serum reduces the coefficient of variation across studies. A second issue arising as we began to compare cohorts was the need to normalize our data between cohorts. Indeed, we determined that a major obstacle to successful integration of cross-cohort data is the diet-independent variability that exists between them (apparently largely biological variability) (Vigneau-Callahan et al., 2001). This effectively requires the identification of a marker that is itself independent of DR in order to normalize the data set. Measurement and subsequent statistical analysis with a two-tailed, unpaired $t$-test of tyrosine concentrations of all the samples in the data set allowed for the determination that tyrosine concentration is not significantly altered by DR. Therefore, quantitation of tyrosine may potentially serve to normalize the concentrations of all metabolites in a given sample.

For normalization purposes, the mean of the tyrosine concentration of each sample in the initial training set was used as the factor to which each individual sample's tyrosine concentration was normalized. This ratio was multiplied by each metabolite's concentration to arrive at a normalized value for that metabolite. Subsequently, the effect of tyrosine normalization was evaluated by documenting any changes in the mean 


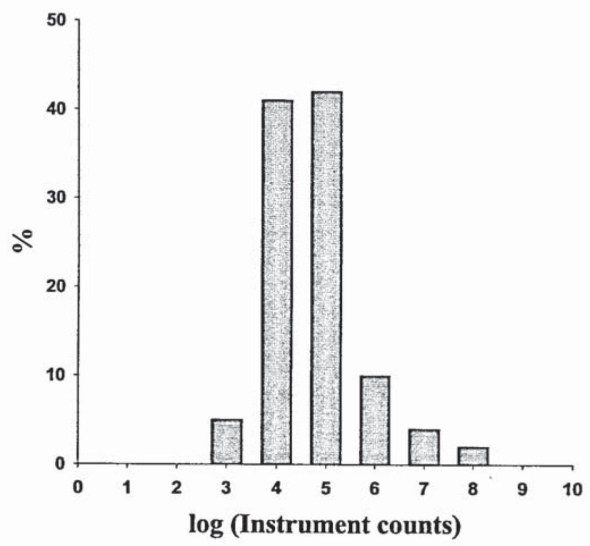

FIG. 3. Biomarker concentrations in sera. The histogram shows percent of total markers broken down by the log of their total current reactivity (a marker of concentration). The biomarker concentrations tend toward a normal distribution, indicating that they may serve as a useful representation of serum metabolites in their entirety.

coefficient of variation of analyte sets sorted either by gender, diet or cohort (Fig. 4). Each column pair shows the coefficients of variation of the entire data set sorted according to a different grouping variable (cohort, gender, or diet). For example, the coefficient of variation was calculated for each metabolite under investigation in the pair labeled "cohort" after sorting the data set according to cohort of origin (i.e., A, $\mathrm{B}$ or C), exclusive of diet or gender. The coefficient of variation of each metabolite was averaged and the averages obtained from the normalized and non-normalized data were compared to determine whether tyrosine normalization reduced variation between cohorts, between genders, or between diets.

The results seem to confirm that normalization to tyrosine concentration will reduce the inter-cohort variability of the sample data set while exerting a nominal effect on the gender and dietary variability.

Cross-gender studies II. The data analysis presented above has simplified subsequent analyses by reducing both the number of metabolites under study and by removing some of their intrinsic variability. We now reinitiate studies of the DR metabolome by initially examining univariate statistics. Univariate statistical analysis of the normalized data set identifies metabolites that are significantly altered by DR. Subsequent to same-gender and cross-gender analysis as well as normalization, our working data set contained values for every metabolite under investigation in both male and female samples, irrespective of the serotype in which the metabolite was originally defined. We used two-tailed, unpaired $t$-tests on each metabolite in our completed data set to evaluate possible significant changes in sera concentration in the DR samples with respect to the AL samples. The $p$-values obtained from the $t$-tests are presented in Table 1. Our results indicate that out of a possible 93 metabolites under investigation, DR significantly altered the concentration of 56 of these in females and 39 in males. This is based on the initial criteria used to minimize Type II statistical errors, $p \leq 0.2$ (Shi et al., 2002b,c).

Cross-gender comparison did serve to identify additional useful metabolites that had been missed previously. Following backwave removal, the female serotype contained 56 metabolites, 31 of which were thought to be of use exclusively in female samples. Statistical analysis of these 31 metabolites in the female samples demonstrated that 18 had a $p \leq 0.2$ between AL and DR (Table 1, data column 2). Additionally, nine 


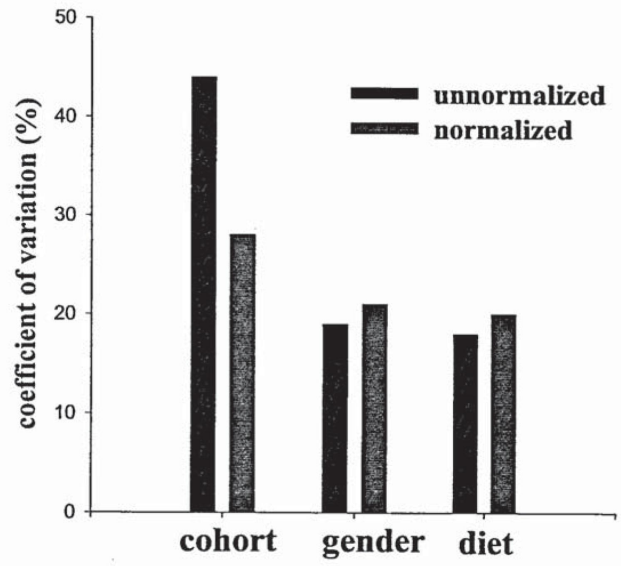

FIG. 4. Normalization against tyrosine corrects inter-cohort differences without altering other differences. These data illustrate the effect that tyrosine-based normalization has on the coefficient of variation of our dataset sorted by cohort, gender or diet. There is a marked reduction in variation between cohorts, while a nominal increase is demonstrated between both gender and diet. Note that this normalization reduces inter-cohort variability to a level comparable to that observed between both gender and diet.

of these 31 metabolites were significantly altered in the male samples after DR (Table 1, data column 4). Following backwave removal, the male serotype contained 62 metabolites, 37 of which were thought to be of use exclusively in male samples. Statistical analysis of these 37 metabolites in the male and female samples demonstrated both contained a total of 18 that had a $p \leq 0.2$ between AL and DR (Table 1, data columns 2 and 5). The female and male serotype shared 25 common metabolites. Statistical analysis of these 25 in female samples found 21 that were significantly altered by DR, while in the male samples only 12 were significantly altered (Table 1, data columns 3 and 6).

Table 1. Metabolites Originally Identified as Part of the Female or Male Serotypes (31, 37 Metaboltres, Respectively) or Both (25 Metaboltres) Were Scored in Male and Female Samples

\begin{tabular}{|c|c|c|c|c|c|c|}
\hline \multirow[b]{3}{*}{ p } & \multicolumn{6}{|c|}{ Ad libitum/diet-restricted $\mathrm{p}$ values } \\
\hline & \multicolumn{3}{|c|}{ Female samples } & \multicolumn{3}{|c|}{ Male samples } \\
\hline & F sero & $M$ sero & $M, F$ sero & $F$ sero & $M$ sero & $M, F$ sero \\
\hline 0.01 & 12 & 10 & 18 & 0 & 2 & 1 \\
\hline 0.05 & 2 & 2 & 0 & 2 & 5 & 3 \\
\hline 0.1 & 3 & 1 & 2 & 1 & 5 & 5 \\
\hline 0.2 & 0 & 5 & 1 & 6 & 6 & 3 \\
\hline 0.5 & 4 & 13 & 1 & 8 & 11 & 5 \\
\hline 1.0 & 10 & 6 & 3 & 14 & 8 & 8 \\
\hline
\end{tabular}

DR significantly altered $56 / 93$ tested metabolites in females and $39 / 93$ in males based on the initial criteria used to minimize Type II statistical errors, $p \leq 0.2$. 


\section{PAOLUCCI ET AL.}

These cross-gender studies also pointed out the complex interactions between gender and diet with respect to the metabolome. The data in Table 1 highlights the extent to which elements of our profile appeared more likely to be truly different (specifically, lower $p$ values) between AL and DR females than between AL and DR males. Both this greater abundance and the relative mean differences across those peaks differing at $p<0.2$ are shown in Figure 1 (top two graphs, mean \pm SEM, listed by, but not scaled to, retention time). Note that this graph also reveals an interesting difference between the effects of DR on males and females. Those metabolites that differ between females on the different diets are almost always present in lower concentration in the DR than the AL rats $(51 / 56)$, whereas this ratio is nearly neutral in males (20/37). The number of metabolites that differ between males and females were found to be greater in $\mathrm{AL}$ than DR rats (60/93 vs. 48/93, the bottom two panels in Figure 5, comparing analytes at $p<0.2,41 / 93$, vs. 15 over 93 at $p \leq 0.01$ ). This graph also reveals an interesting difference between the effects of DR on those metabolites that differ between the sexes. Those peaks that differ between males and females on the AL diets are almost always present in higher concentrations in the female rats $(56 / 60)$, whereas this ratio is neary neutral in the DR rats (26/48). These differences are presented by $p$ value in Figure 6.

These data reveal that it will be necessary to build independent mathematical models to reflect the serotypes (metabolomes) of males and females subjected to DR.

General implications for metabolomic studies. The work presented in this report addresses three areas of our study that are of general interest in the study of metabolome-nutrition interactions. These questions all center around the conversion from single cohort datasets to datasets that can address questions across the entire populations that we study. These areas may be summarized as analytical concerns, gender-specific metabolic profiles, and the requirement for examination of profiles vs single metabolites.

The first area concerns the analytical issues involved in conducting a high data density study of samples that are collected over time for study of interactions between nutrition and the metabolome. As seen in this report, the practice of validating multiple independent sets of metabolites, initially described in previous papers (Vigneau-Callahan et al., 2001; Shi et al., 2002b,c), increases the yield of informative metabolites without increasing noise to levels that impair analysis. Our analysis also identified the importance of finding a metabolite/variable that can function for normalization. Surprisingly, this task was relatively complex, as the concentration of most metabolites examined varyed significantly across different cohorts. Fortunately, tyrosine appeared to serve well as a normalizing agent. Third, our work suggests that any study of the interaction between nutrition and the metabolome must consider a deliberate multicohort design to avoid biases introduced by the use of a single cohort.

The second area concerns the comparative effects of DR on males and females. Although gender-specific effects in DR are well-established, the major effects of DR-such as increased longevity and decreased morbidity - are highly consistent between males and females (in most strains). This leads to a straight-forward hypothesis to be tested: DR modulates the metabolome of male and female animals equivalently. Other, weaker hypotheses that can be addressed in this area are that most metabolites will be well-conserved, the general trends will be well-conserved, and that the major changes will be well-conserved between genders. The data presented in this paper argue against each of these, suggesting that the metabolome undergoes fundamentally gender-specific changes, with comparatively few gender-independent changes. In the accompanying paper (Paolucci et al., 2004) we will test a related hypothesis--whether classification based approaches can separate male and female AL and DR simultaneously.

The third area concerns the power and utility of megavariate-based analysis for the study of metabolomenutrient interactions. This utility is reflected in two complementary ways. First, less than half of the analytes that comprise our serotypes would be considered to vary significantly between groups by classical criteria $(p<0.05)$. Furthermore, many of those that do meet these criteria would not have been identified if we had not been able to work with the weaker criteria initially established $(p<0.2)$. The use of multi- $I$ mega-variate approaches (HCA, PCA) enabled us to identify profiles that worked, and carry the analytes that comprised them forward, with minimal concern about false positives. Thus, we have essentially identified a tool that considerably increases the statistical power of metabolomics studies. Second, even those metabolites that display statistically significant differences show considerable overlap between the two 

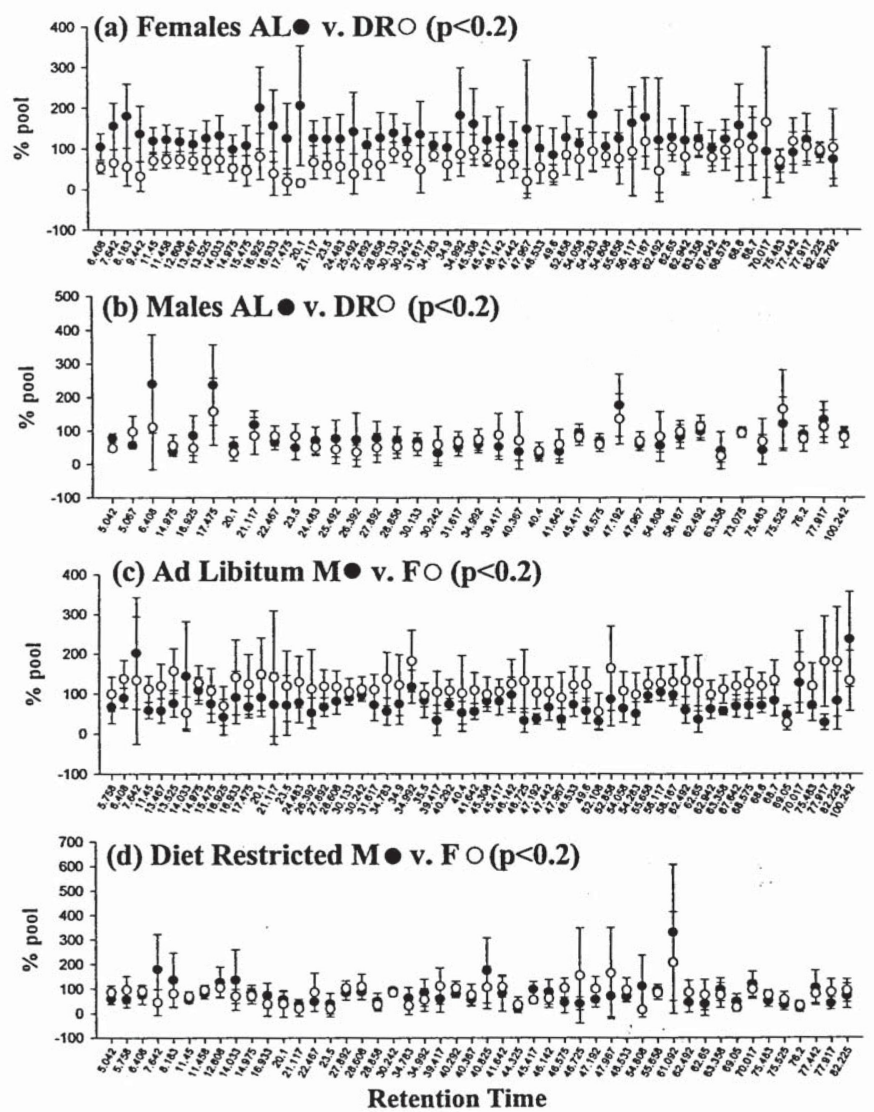

FIG. 5. Gender-diet interactions-metabolite concentrations relative to pools. The mean \pm sem (as percent of the standard pool) for all metabolites with $p$ values of $<0.2$ between $A L$ and DR female rats (top); AL and DR male rats ( $2^{\text {nd }}$ down); male and female AL rats ( $3^{\text {rd }}$ down); and male and female DR rats (bottom). Retention time is used as qualitative label rather than as a continuous quantitative variable to more clearly present mean $\pm S E M$.

groups. Thus, univariate statistical approaches in this system (AL-DR) do not identify class distinguishing elements (sometimes termed state-markers-markers whose presence or absence essentially defines the existence of a condition), while megavariate based approaches readily do so. From a conceptual viewpoint, this may be considered in following way: Visual inspection of the data in Figure 5 suggests an obvious pat- 


\section{PAOLUCCI ET AL.}
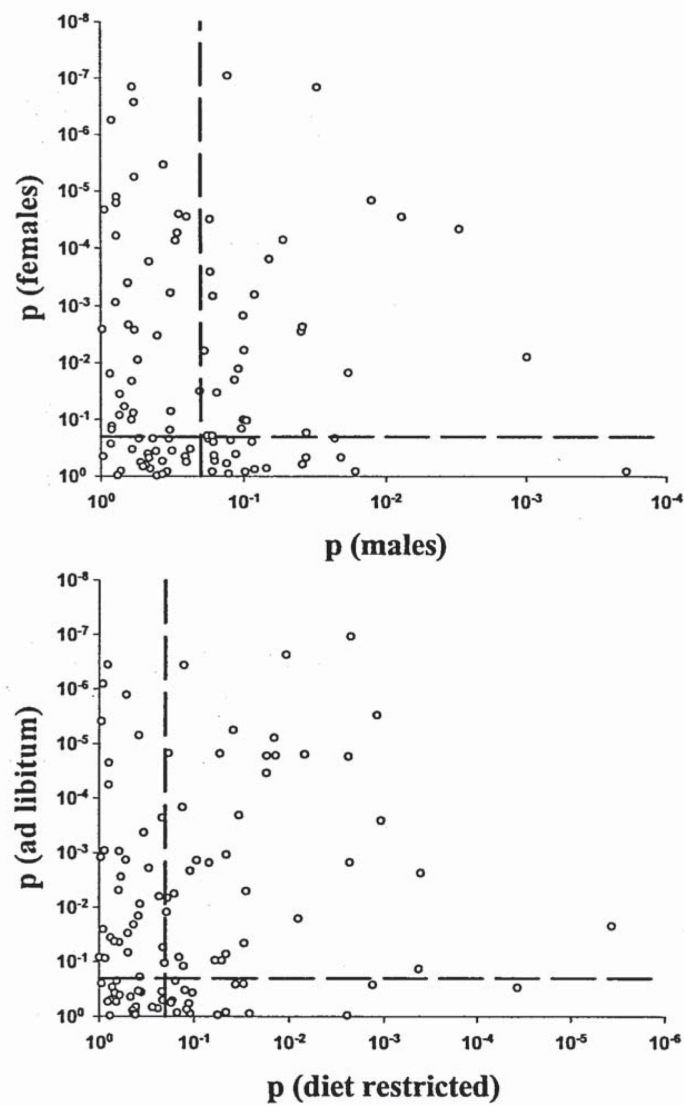

FIG. 6. Gender-diet interactions- $-p$ value distributions. Metabolites in the profile are plotted by: $p$ values between $\mathrm{AL}$ and DR in males versus females (top); $p$ values between males and females in AL and DR (bottom). Each plot is divided into four quadrants by the intersection of lines drawn at $p=0.2$ on each axis.

tern difference between AL and DR-but in large part this is obvious because humans recognize patterns so well. Mathematical identification, validation, and re-recognition of this pattern is often difficult, but we have accomplished this goal, as described in the previous and subsequent reports (Vigneau-Callahan et al., 2001; Shi et al., 2002b,c, 2004; Paolucci et al., 2004). 


\section{DIET METABOLIC SEROTYPES-CROSS-COHORT STUDIES}

\section{CONCLUSION}

This report furthers our attempts to generate potential biomarkers of DR by integrating the data across cohorts and by conducting cross-gender comparisons. Tyrosine-based normalization of the data set has permitted corrective scaling of our raw sample scores in order to compensate for potential variability in individual chromatogram peak intensities. Elimination of back-waves was the final step in reducing the size of our data set before applying statistical analysis for validation across cohorts. Conducting cross-gender studies revealed a set of metabolites that had been identified in only one gender previously, but which indeed do differ statistically in both genders. Thus, overall, 56 and 39 of 93 variables differ at our cutoff of $p<0.2$ in females and males, respectively. We further show that DR appears to have greater effects on the metabolome in females as compared to that of males, and that DR appears to reduce inter-gender differences in the metabolome. Most importantly, the analyses presented compress our six previous datasets (three cohorts each of females and males with 63 and 66 variables, respectively) into a single, 93 variable dataset by collapsing cohort distinctions, eliminating redundancy, and eliminating backwaves. These data are now in an appropriate form to allow us to begin to build more powerful mathematical class descriptors and class predictors. These data also present a univariate approach toward pruning this dataset, which will be compared with that derived from multivariate analysis (Paolucci et al., 2004).

\section{ACKNOWLEDGMENTS}

We thank Dr. Tom Vogl for his many critical discussions and comments on the manuscript. This work was supported by NIH NIA R01-AG15354 (B.S.K.), ESA, Inc., and the Winifred Masterson Burke Relief Foundation.

\section{REFERENCES}

ERIKSSON, L., JOHANSSON, E., KETTANEH-WOLD, N., et al. (2001). Multi- and Megavariate Analysis (Umea, Sweden, Umetrics).

FERNANDES, G., CHANDRASEKAR, B., TROYER, D.A., et al. (1995). Dietary lipids and calorie restriction affect mammary tumor incidence and gene expression in mouse mammary tumor virus/v-Ha-ras transgenic mice. Proc Natl Acad Sci USA 92, 6494-6498.

IWASAKI, K., GLEISTER, C.A., MASORO, E.J., et al. (1988). Influence of the restriction of individual dietary components on longevity and age-related disease of Fischer Rats: the fat component and the mineral component. J Gerontol 43, B13-B21.

KENNEDY, R.L., LEE, Y., VAN ROY, B., et al. (1998). Solving Data Mining Problems Through Pattern Recognition (Upper Saddle River, NJ, Prentice Hall).

KLURFELD, D.M., WEBER, M.M., and KRITCHEVSKY, D. (1987). Inhibition of chemically induced mammary and colon tumor promotion by caloric restriction in rats fed increased dietary fat. Cancer Res 47, 2759-2762.

KRISTAL, B.S., VIGNEAU-CALLAHAN, K.E., and MATSON, W.R. (1998). Simultaneous analysis of the majority of low-molecular-weight, redox-active compounds from mitochondria. Anal Biochem 263, 18-25.

KRISTAL, B.S., VIGNEAU-CALLAHAN, K.E., and MATSON, W.R. (2002). Simultaneous analysis of multiple redox-active metabolites from biological matrices. In Methods in Molecular Biology, Vol. 186: Oxidative Stress Biomarkers and Antioxidant Protocols. (Totowa, Humana Press), pp. 185-194.

KRISTAL, B.S., and YU, B.P. (1994). Aging and its modulation by dietary restriction. In Modulation of Aging Processes by Dietary Restriction, 1st ed. B.P. Yu, eds. (Boca Raton, FL, CRC Press), pp. 1-36.

KRITCHEVSKY, D., WEBER, M.M., and KLURFELD, D.M. (1984). Dietary fat versus caloric content in initiation and promotion of 7,12-dimethylbenz(a)anthracene-induced mammary tumorigenesis in rats. Cancer Res 44, $3174-3177$.

MAEDA, H., GLEISER, C.A., MASORO, E.J., et al. (1985). Nutritional influences on aging of Fischer 344 rats: II. Pathology. J Gerontol 40, 671-688.

MANLY, B.F.J. (2000). Multivariate Statistical Methods (Boca Raton, FL, CRC Press). 


\section{PAOLUCCI ET AL.}

MATSON, W.R., GAMACHE, P.H., BEAL, M.F., et al. (1987). EC array sensor concepts and data. Life Sci 41, 905-908.

MATSON, W.R., LANGIALS, P., VOLICER, L., et al. (1984). n-electrode three dimensional liquid chromatography with electrochemical detection for determination of neurotransmitters. Clin Chem 30, 1477-1488.

PAOLUCCI, U., VIGNEAU-CALLAHAN, K.E., SHI, H., et al. (2004). Development of biomarkers based on diet-dependent metabolic serotypes: characteristics of component-based models of metabolic serotypes. OMICS 8, $221-238$.

SHI, H., VIGNEAU-CALLAHAN, K.E., MATSON, W.R., et al. (2002a). Attention to relative response across sequential electrodes improves quantitation of coulometric array. Anal Biochem 302, 239-245.

SHI, H., VIGNEAU-CALLAHAN, K.E., SHESTOPALOV, A.I., et al. (2002b). Characterization of diet-dependent metabolic serotypes: primary validation of male and female serotypes in independent cohorts of rats. $\mathbf{J}$ Nutr $\mathbf{1 3 2}$, 1039-1046.

SHI, H., VIGNEAU-CALLAHAN, K.E., SHESTOPALOV, A.I., et al. (2002c). Characterization of diet-dependent metabolic serotypes: proof of principle in female and male rats. J Nutr 132, 1031-1038.

SHI, H., PAOLUCCI, U., VIGNEAU-CALLAHAN, K.E., et al. (2004). Development of biomarkers based on diet-dependent metabolic serotypes: practical issues in development of expert system-based classification models in metabolomic studies. OMICS 8, 197-208.

SHIMOKAWA, I., HIGAMI, Y., YU, B.P., et al. (1996). Influence of dietary components on occurrence of and mortality due to neoplasms in male F344 rats. Aging Milano 8, 254-262.

VIGNEAU-CALLAHAN, K.E., SHESTOPALOV, A.I., MILBURY, P.E., et al. (2001). Characterization of diet-dependent metabolic serotypes: analytical and biological variability issues in rats. J Nutr $924 \mathrm{~S}-932 \mathrm{~S}$.

WEINDRUCH, R., and WALFORD, R. (1988). The Retardation of Aging and Disease by Dietary Restriction (St. Louis, Charles C. Thomas).

WILLETT, W.C., DIETZ, W.H., and COLDITZ, G.A. (1999). Guidelines for healthy weight. N Engl J Med 341, $427-434$.

YU, B.P. (1994). Modulation of Aging Processes by Dietary Restriction (Boca Raton, FL, CRC Press).

Address reprint requests to:

Dr. Bruce S. Kristal

Dementia Research Service

Burke Medical Research Institute

785 Mamaroneck Ave.

White Plains, NY 10605

E-mail: Bkristal@burke.org 


\section{GENERAL INFORMATION}

OMICS A Journal of Integrative Biology is a peer-reviewed journal covering all aspects of integrative biology, diverse "OMICS-es," such as genomics, proteomics, pharmacogenomics, etc., and their integration, data analysis, and modeling.

OMICS A Journal of Integrative Biology (ISSN: 1536-2310) is published 4 times a year (quarterly) by Mary Ann Liebert, Inc., 2 Madison Avenue, Larchmont, NY 10538-1962. Postmaster: Send address changes to OMICS A Journal of Integrative Biology, c/o Subscription Department, Mary Ann Liebert, Inc., 2 Madison Avenue, Larchmont, NY 10538-1961. Mailed in Canada under CPM \#40026674.

Subscriptions should be addressed to the Publisher and are payable in advance. Rates for subscriptions are for a volume of 4 issues: USA print $\$ 634$, international print $\$ 788$, USA print and online $\$ 753$, international print and online $\$ 910$, and online only (worldwide) $\$ 586$. Subscriptions begin with the first issue of the current volume. Bulk subscriptions are available upon request from the Publisher. No cancellations/refunds can be honored after publication of a volume's first issue and no refunds/returns can be honored on single issue purchases.

OMICS A Journal of Integrative Biology is owned and published by Mary Ann Liebert, Inc. Copyright ${ }^{\circ} 2004$ by Mary Ann Liebert, Inc. Printed in the United States of America.

Telephone: (914) 834-3100; fax: (914) 834-1388; e-mail: info@ liebertpub.com Online: www.liebertpub.com

Information for Manuscript Submission is given in the back of this issue and on our website: www. liebertpub.com

Business communications should be addressed to the Publisher.

Advertising inquiries from within the United States should be addressed to Catherine Hiller, Mary Ann Liebert, Inc., 2 Madison Avenue, Larchmont, NY 10538-1961. Telephone: (914) 834-3100. For Europe/Outside the U.S., contact: Hilary Turnbull, imPRESS International Media Ltd., Carrington Kirk, Carrington, Midlothian, EH23 4LR, UK. Telephone: +44-(0)-1875-825700; fax: +44-(0)-1875-825701; e-mail: impress@impressmedia.com All advertisements are subject to approval by the Publisher.

Reprints, except special orders of 100 or more, are available from the authors. For permission to photocopy 24 copies or fewer of an article for internal purposes, please request permission and pay the appropriate fee by contacting the Customer Relations Dept. of the Copyright Clearance Center, Inc., 22 Rosewood Drive, Danvers, MA 01923. Telephone: (978) 750-8400; fax: (978) 750-4470. If the number of copies of an article is 25 or more, contact the Reprint Sales Manager, Karen Ballen, directly at (914) 834-3100, ext. 609 , for options.

Manuscripts should be directed to the Editor-in-Chief: Eugene Kolker, Ph.D., BIATECH, 19310 North Creek Parkway, Suite 115, Bothell, WA 98011. Telephone: (425) 481-4590; fax: (425) 481-5384; e-mail: ekolker@biatech.org.

All papers, news, comments, opinions, findings, conclusions, or recommendations in OMICS A Journal of Integrative Biology are those of the author(s), and do not constitute opinions, findings, conclusions, or recommendations of the Journal, its Publisher, and its editorial staff.

OMICS A Journal of Integrative Biology is indexed in Index Medicus/MEDLINE and Cambridge Scientific Abstracts.

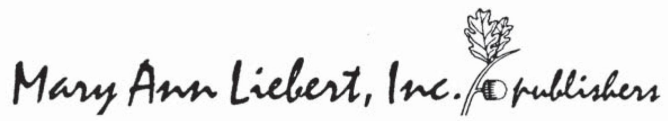

www.liebertpub.com 